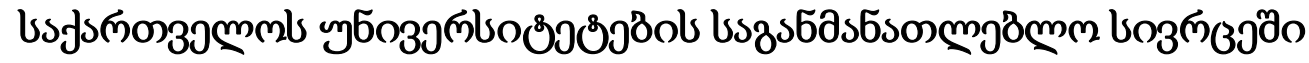

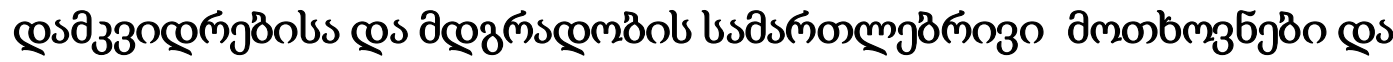

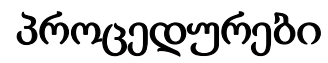

\author{
зозомsдазомо бобм

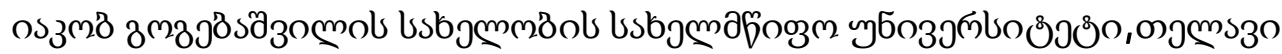

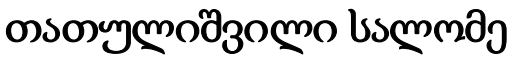

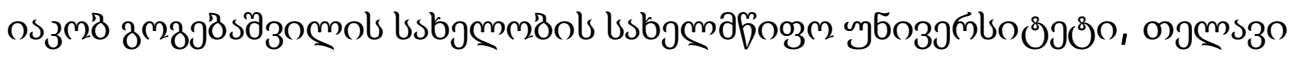

https://doi.org/10.52340/idw .2021.537

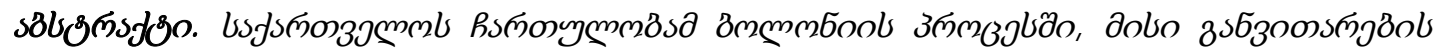

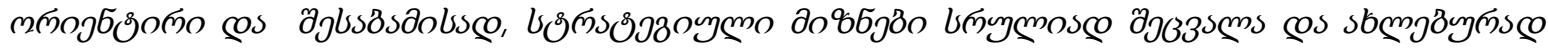

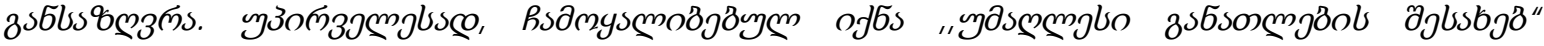

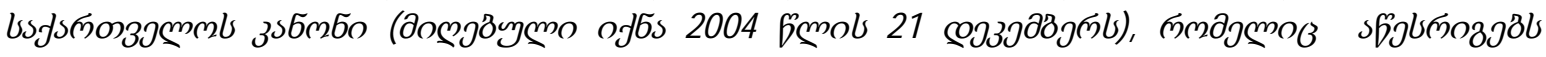

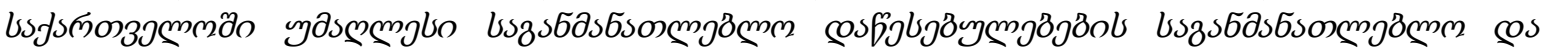

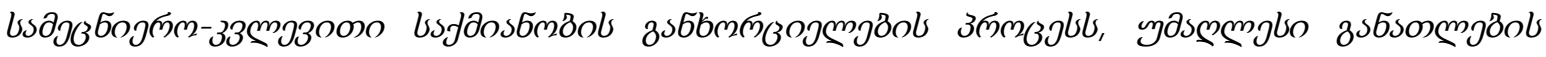

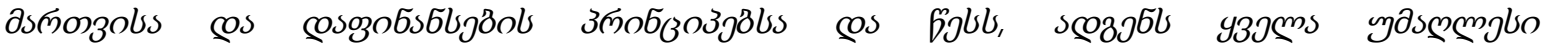

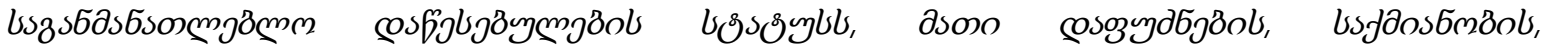

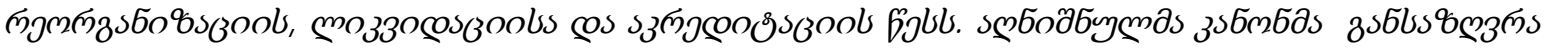

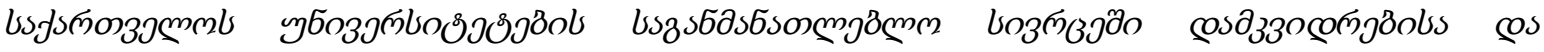

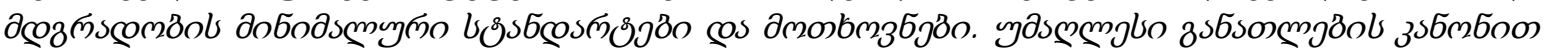

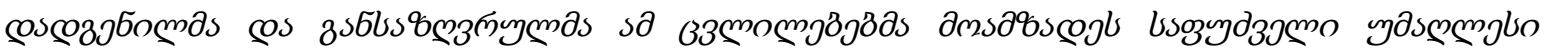

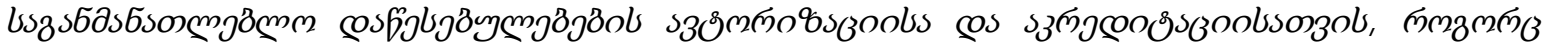

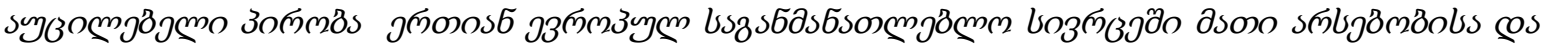

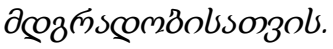

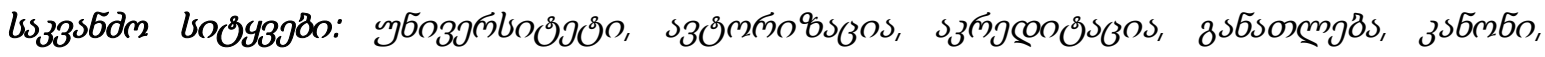
поомп्丶

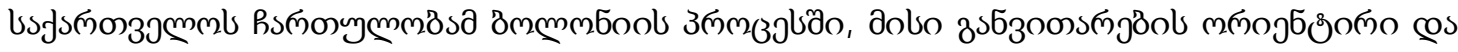

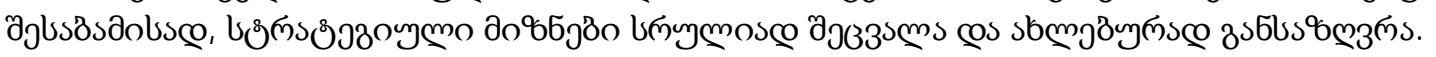

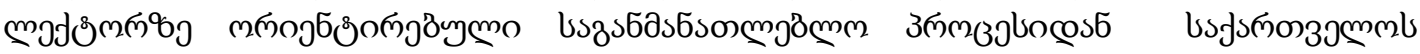

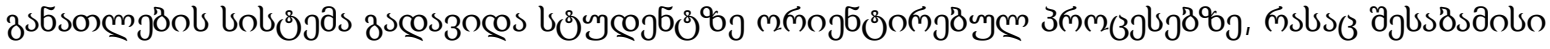

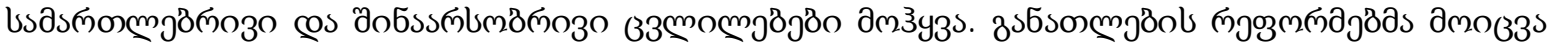

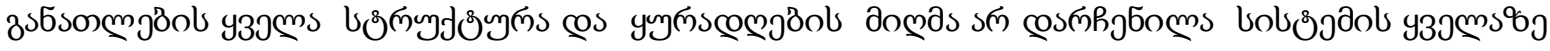

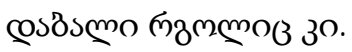

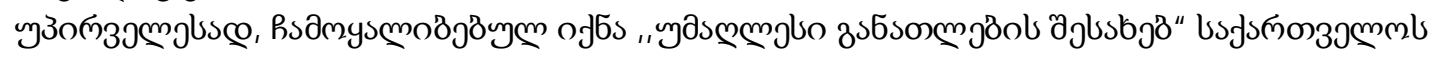

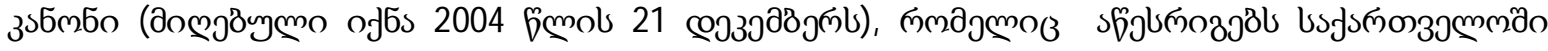

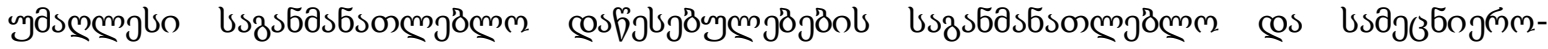

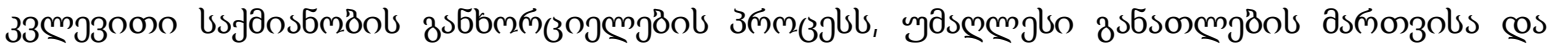

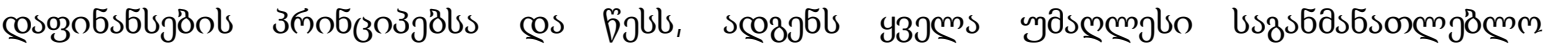

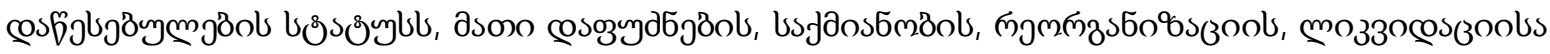
cos sз̆ 


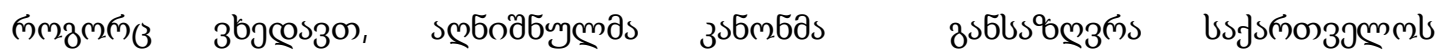

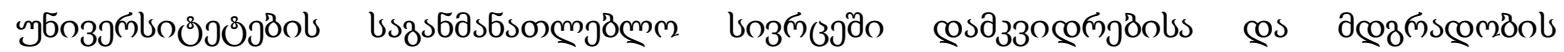

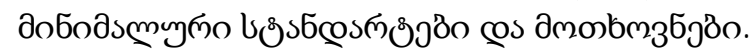

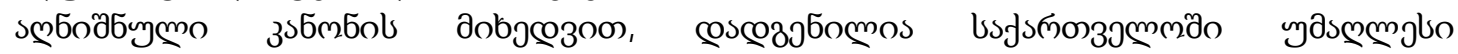

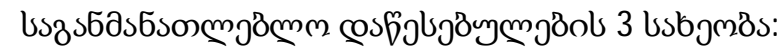

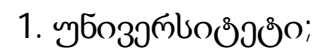

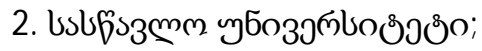

3. зलмлगхо.

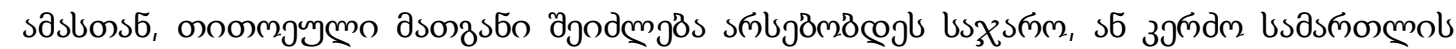

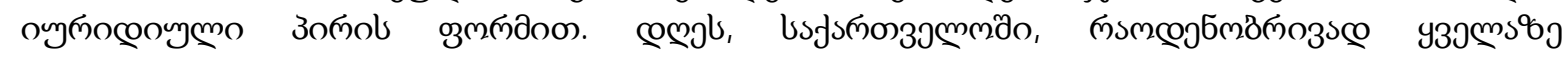

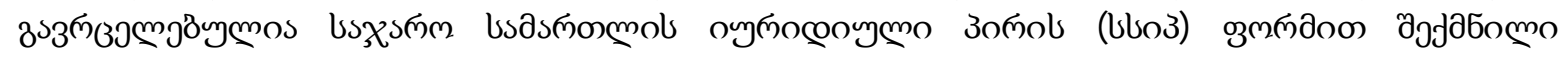

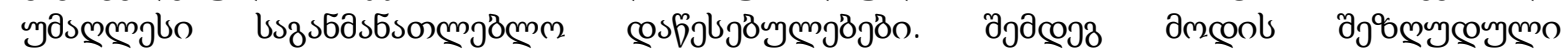

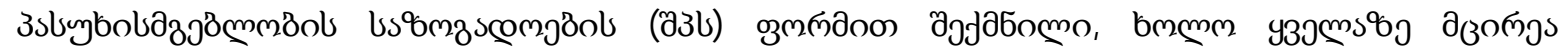

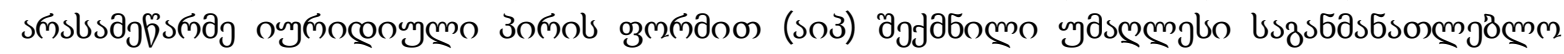

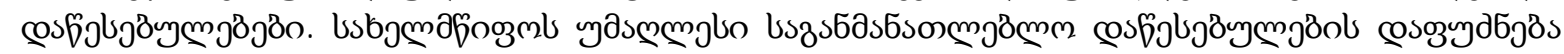

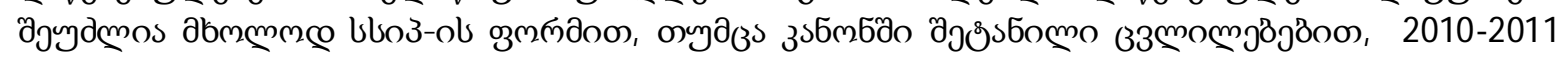

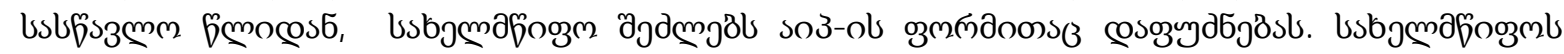

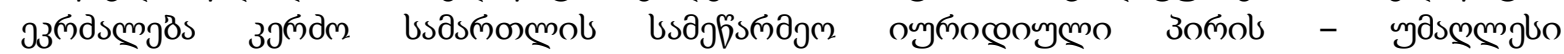

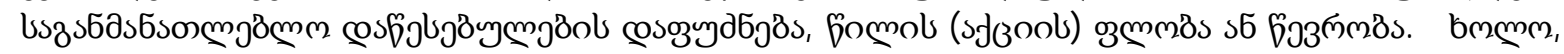

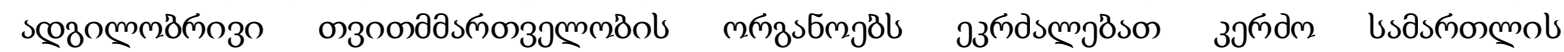

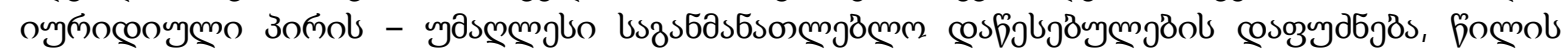

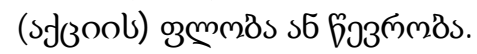

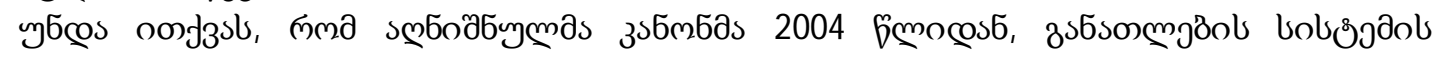

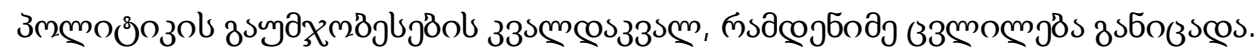

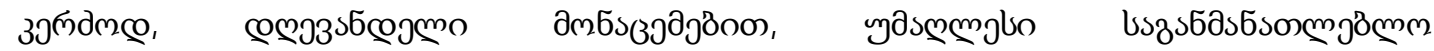

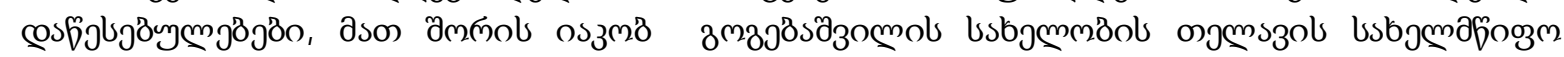

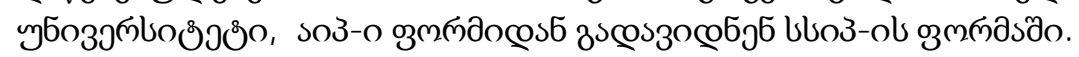

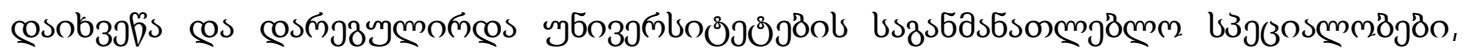

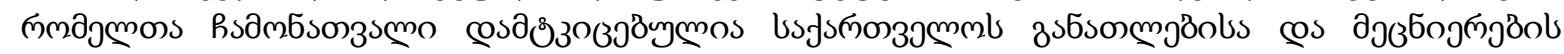

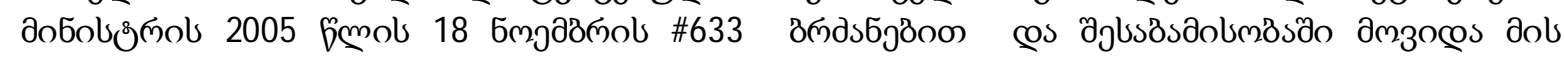

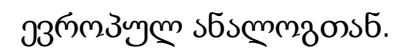

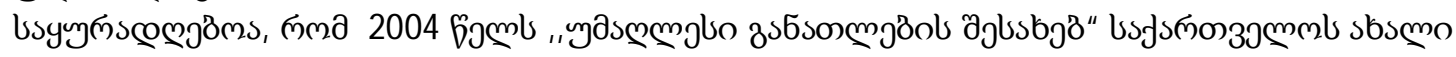

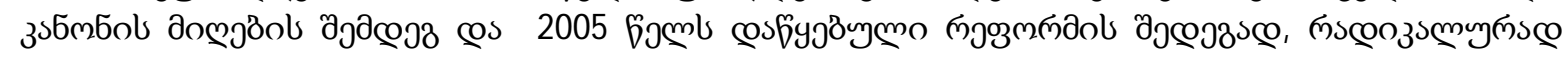

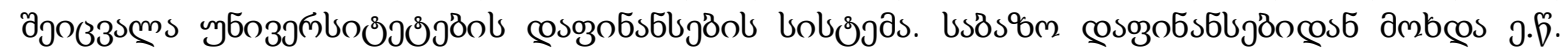

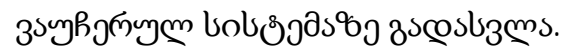

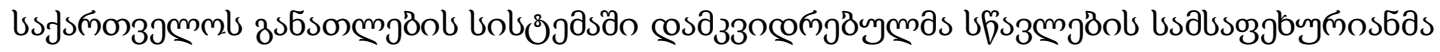

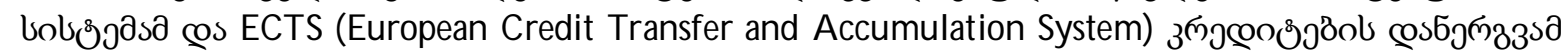

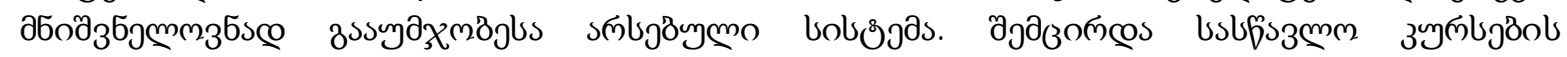

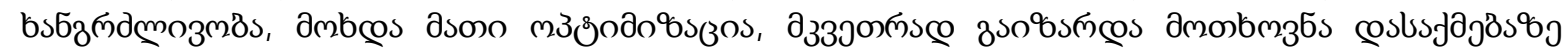

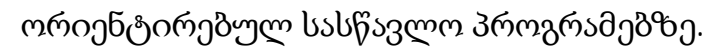

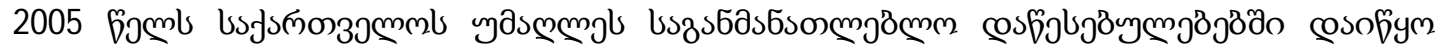

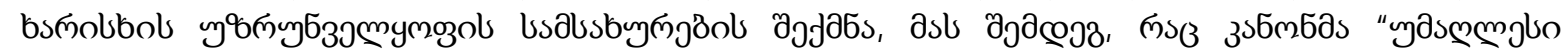

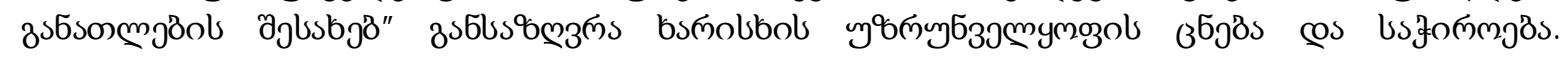

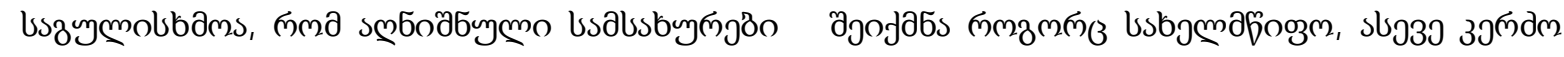

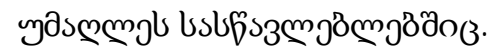

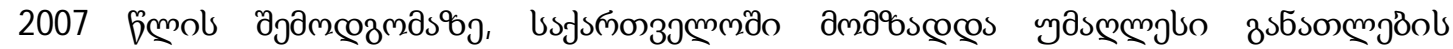

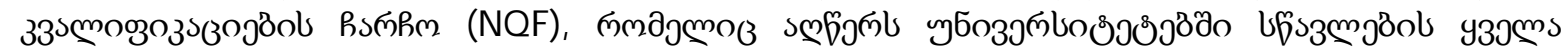

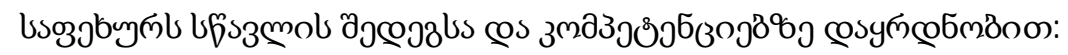

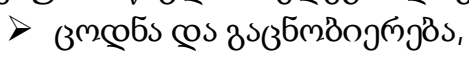

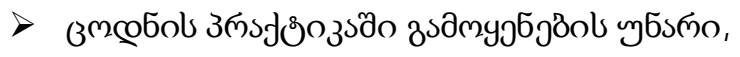




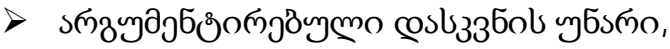

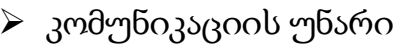

$>$ uf́s 3 mols y6s

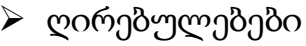

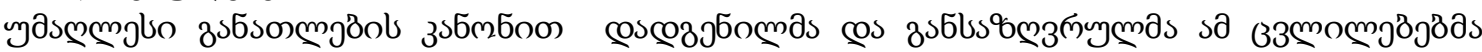

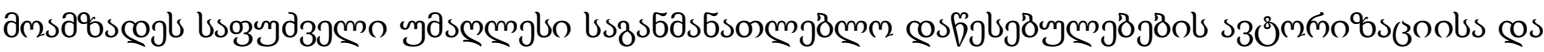

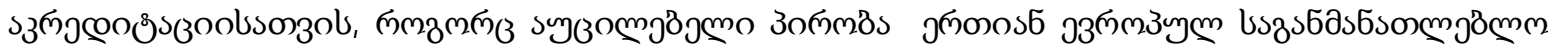

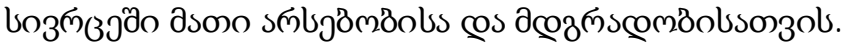

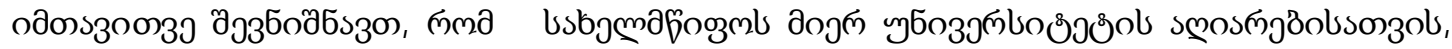
убозэкио

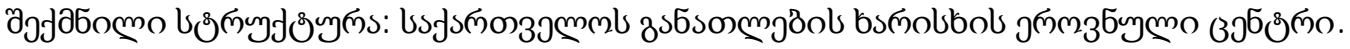

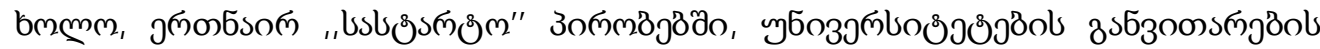

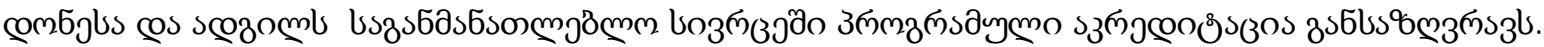

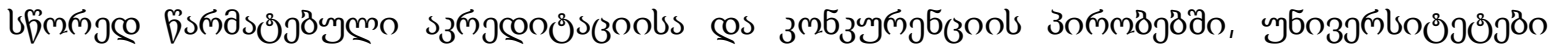

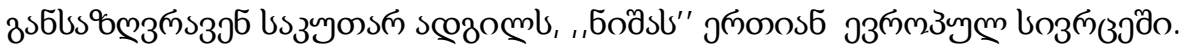

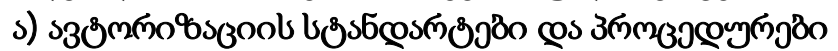

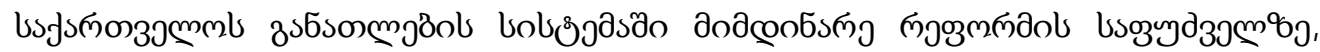

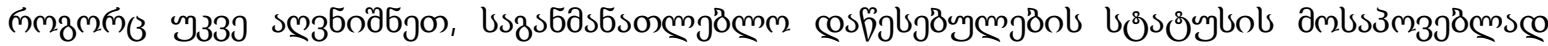

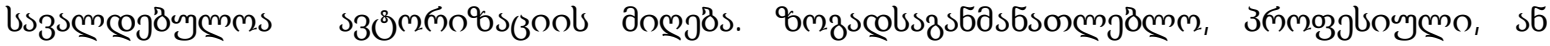

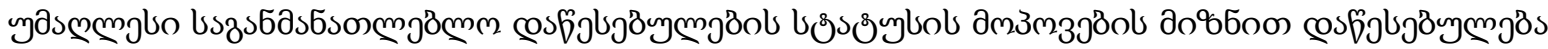

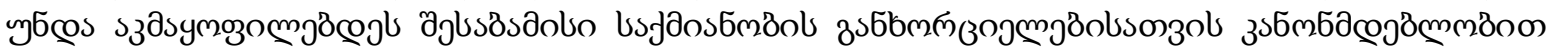

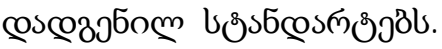

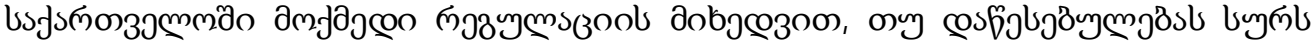

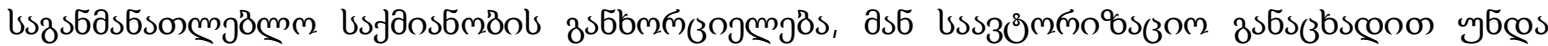

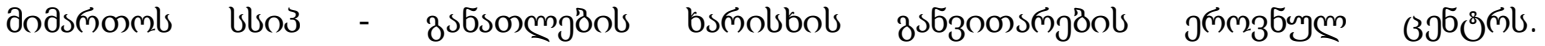

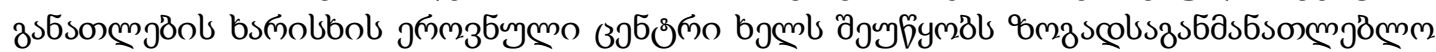

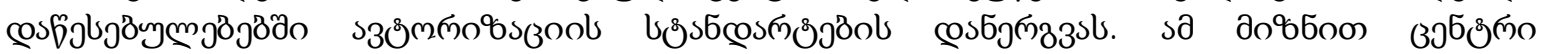

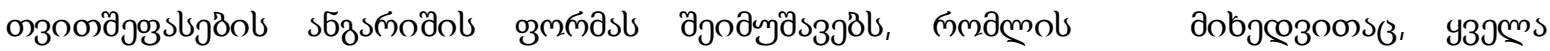

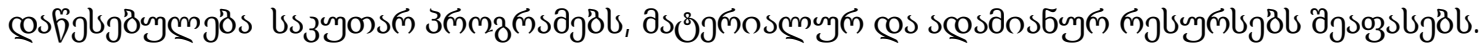

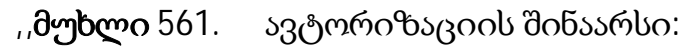

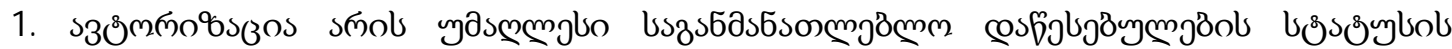

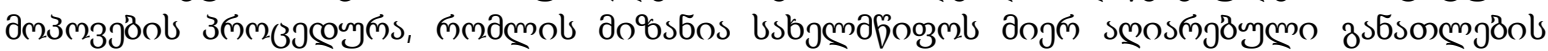

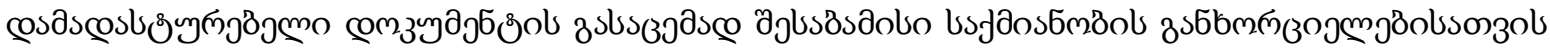

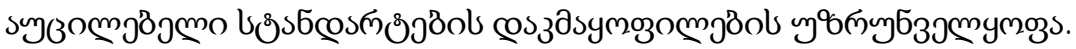

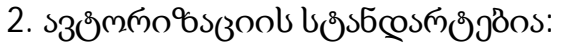

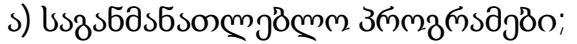

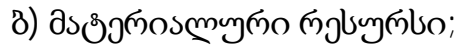

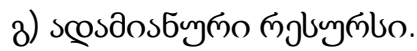

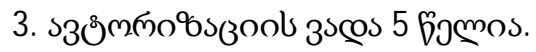

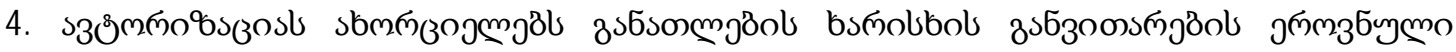

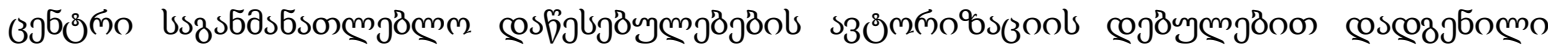

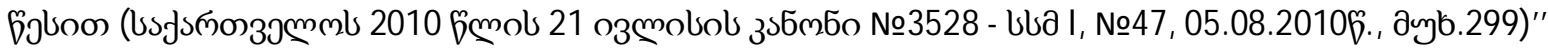

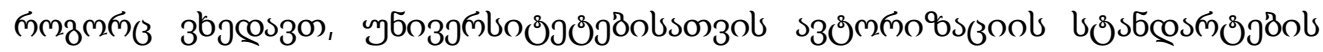

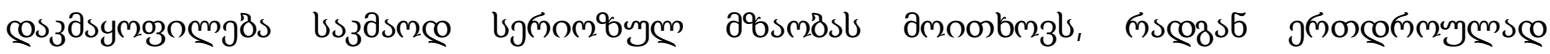

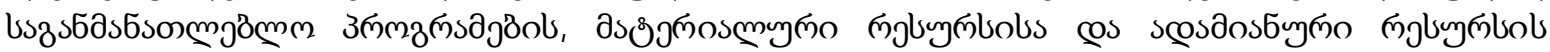

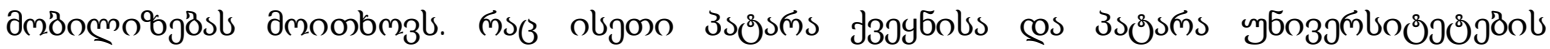

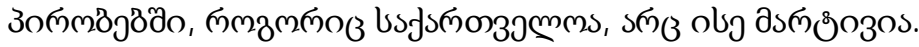

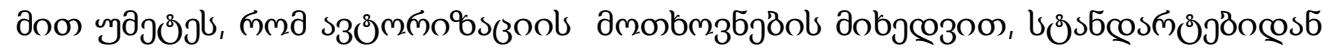

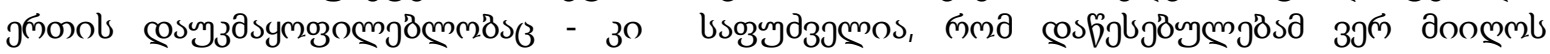

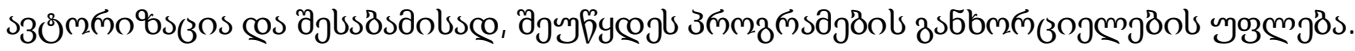




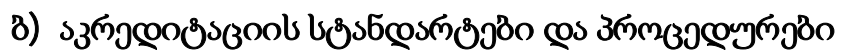

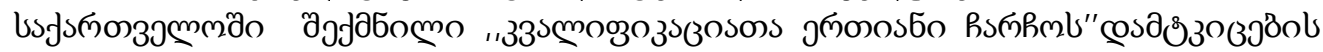

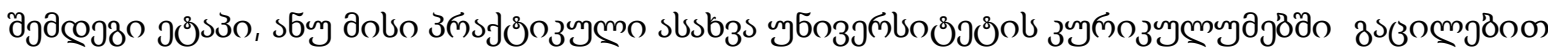

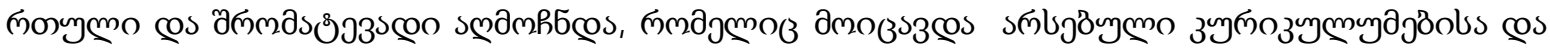

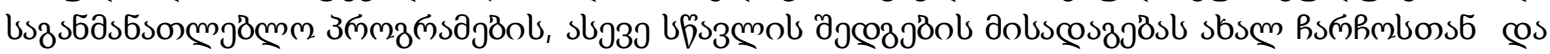

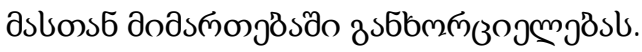

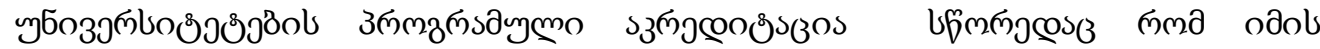

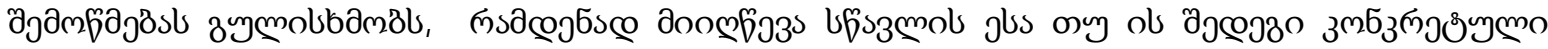

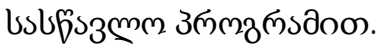

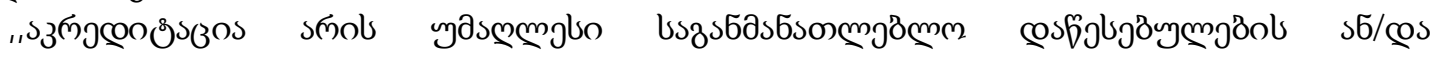

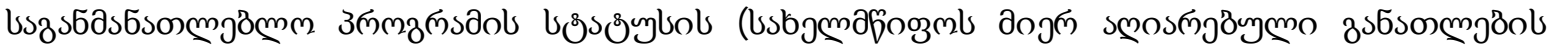

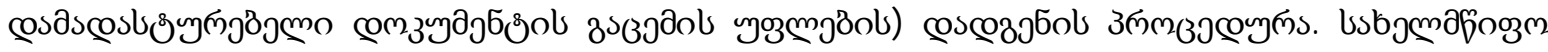

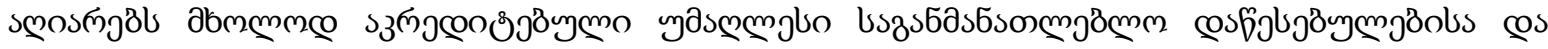

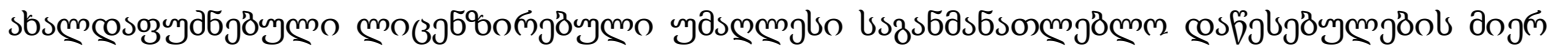

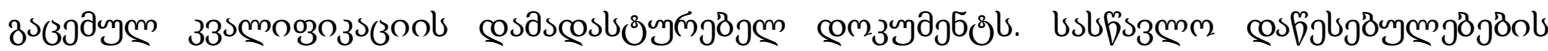

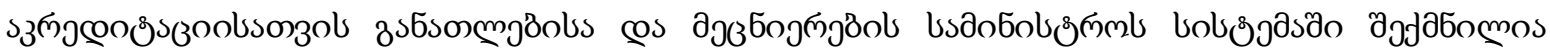

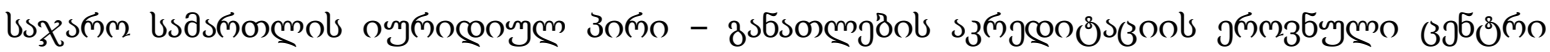
(www. Eqe.ge)".

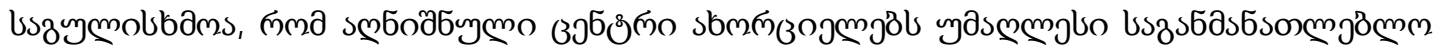

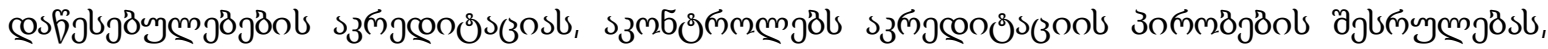

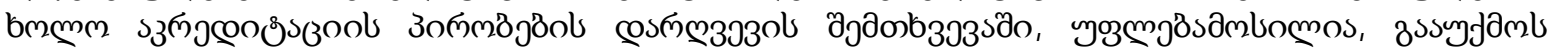

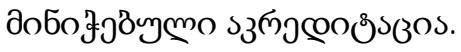

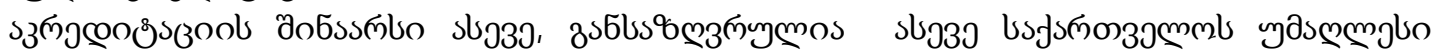

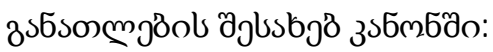

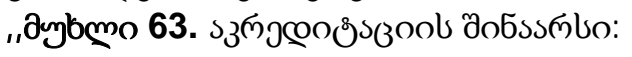

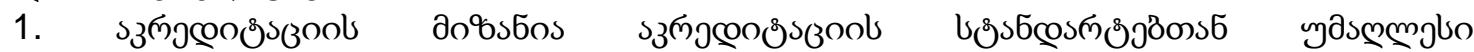

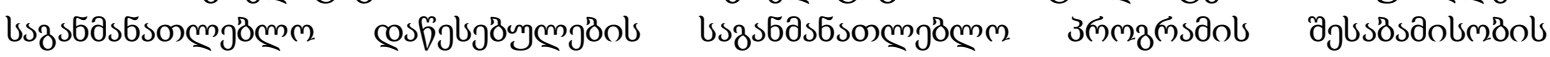

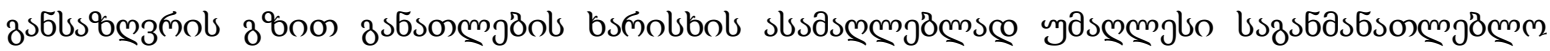

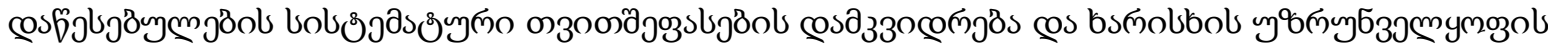

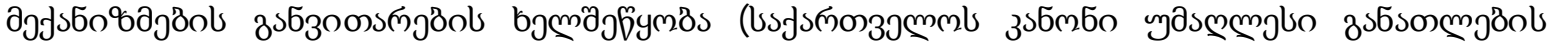
'jglsbjo, 2004)".

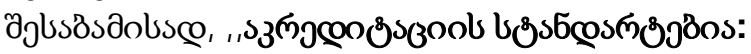

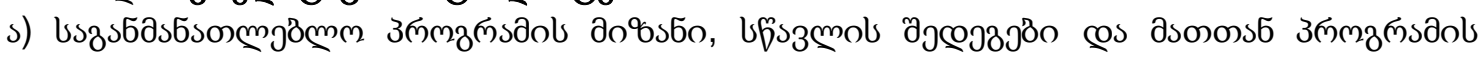
aglsossaobm8s;

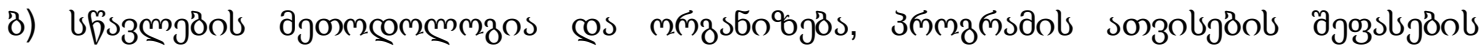

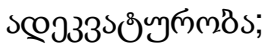

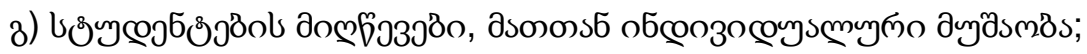

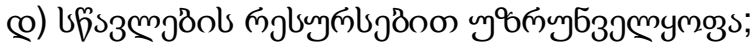

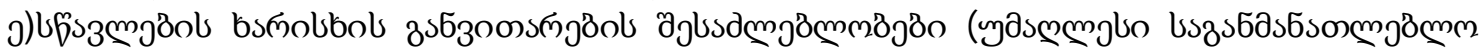

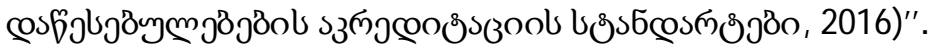

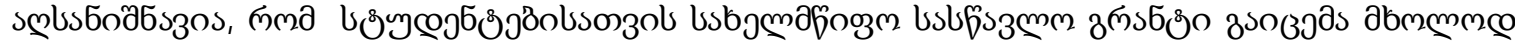

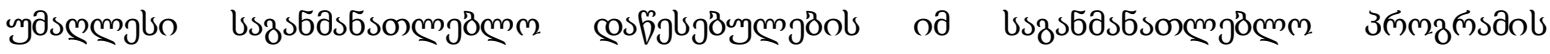

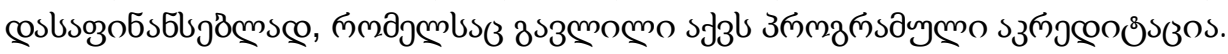

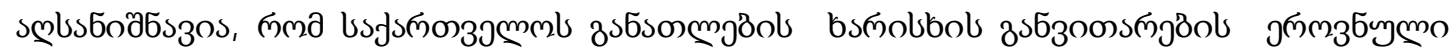

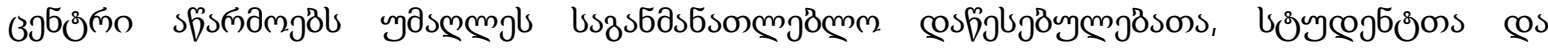

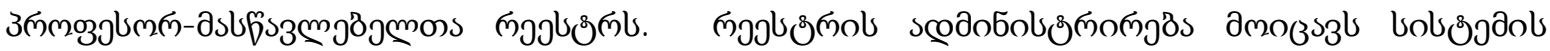

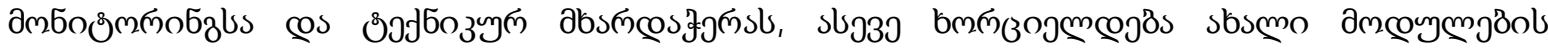
उलмэдठ욯․

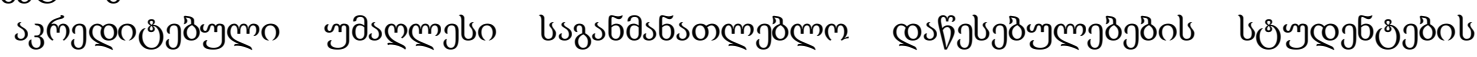

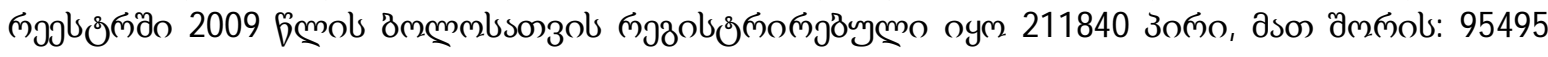




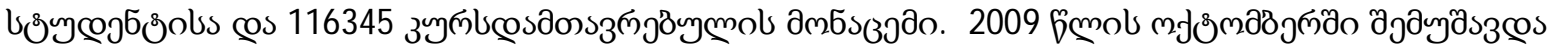

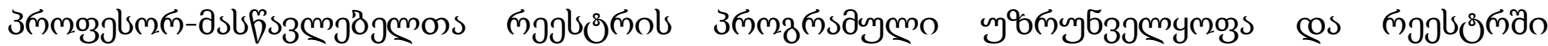

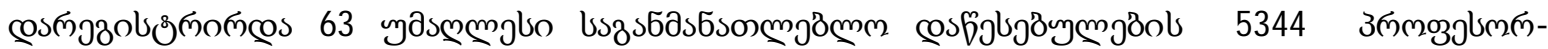

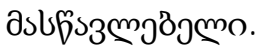

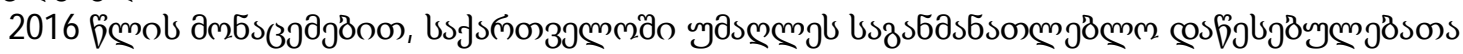

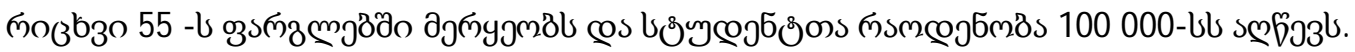

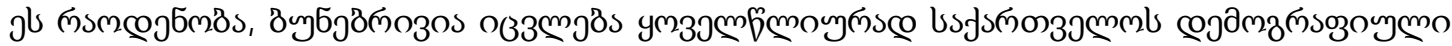

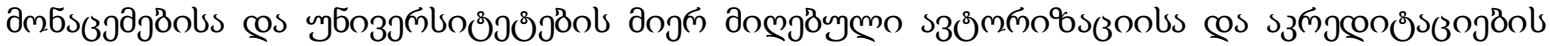
дobjezon.

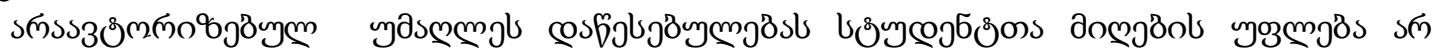
zssh6os.

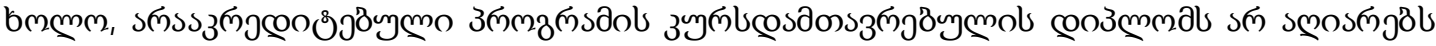

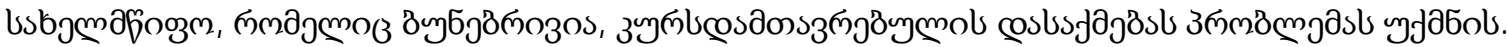

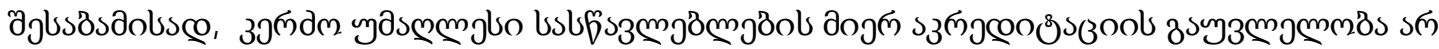

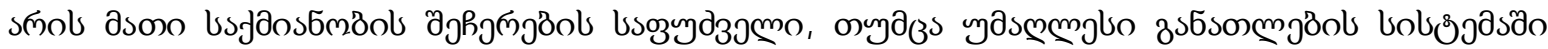

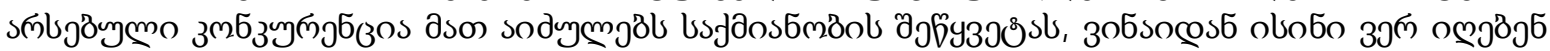

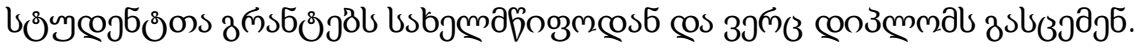

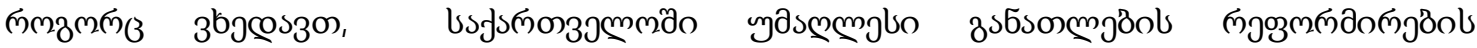

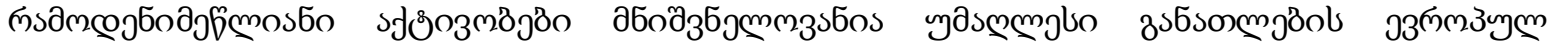

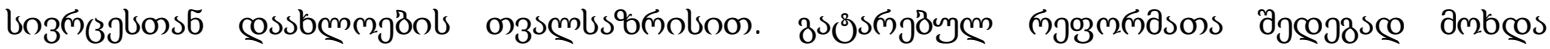

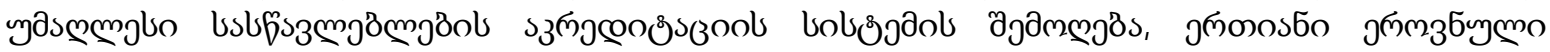

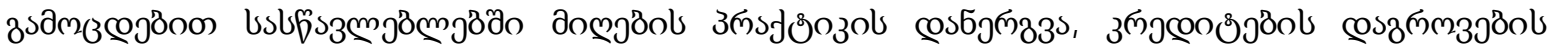

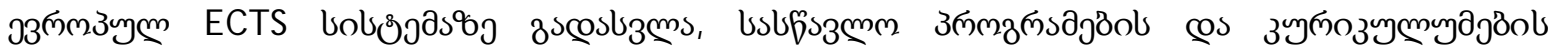

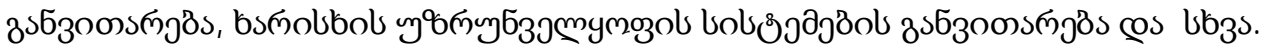

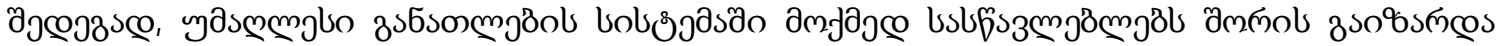

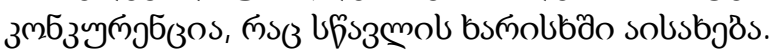

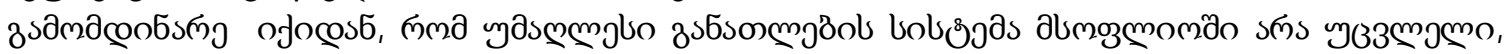

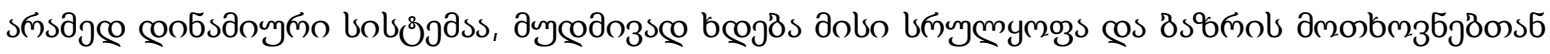

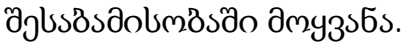

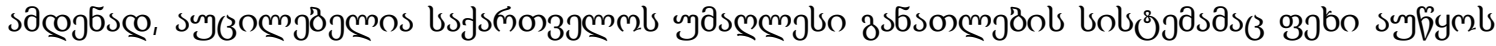

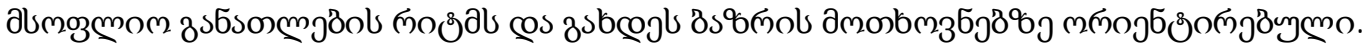

пмдмпп З

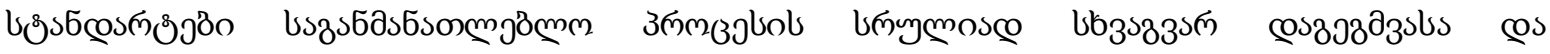

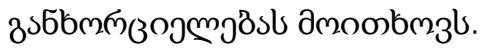

sf astusozs

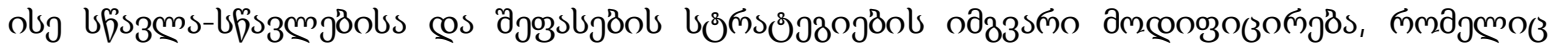

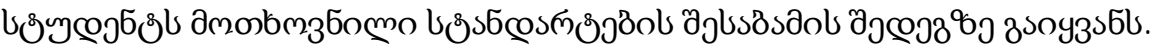

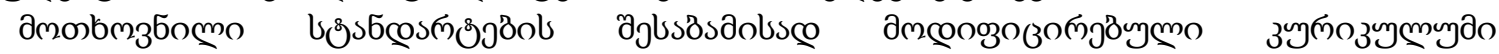

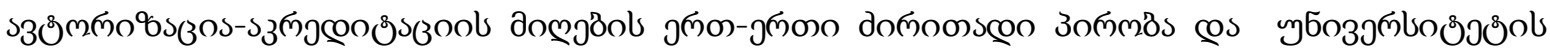

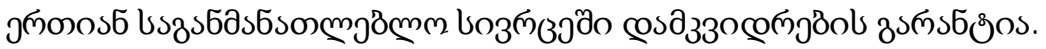

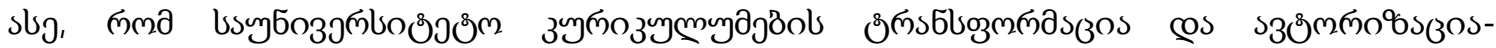

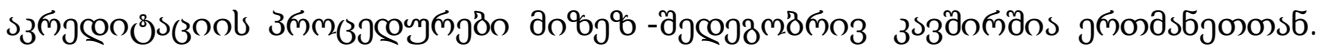

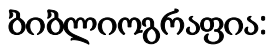

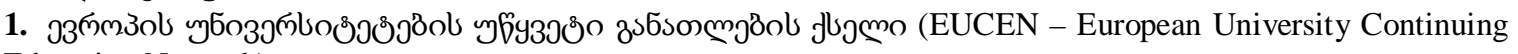
Education Network)

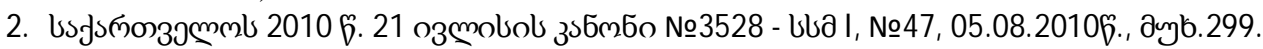

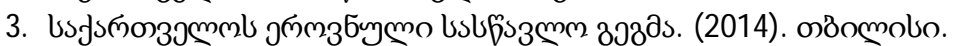

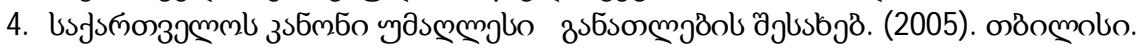




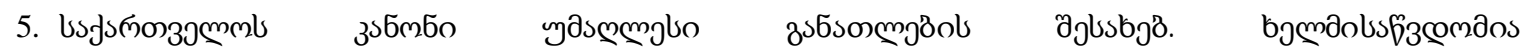
https://matsne.gov.ge/ka/document/view/32830\%23

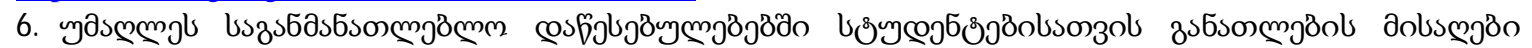

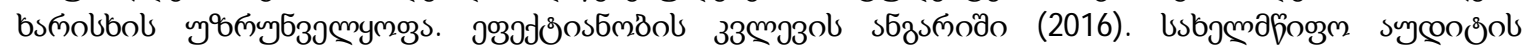
usabsbigno. bjemaobsficomaos:

http://sao.ge/files/auditi/auditis-angarishebi/2016/usd-ganaTlebis-misaRebi-xarisxi.pdf

7. эдsме

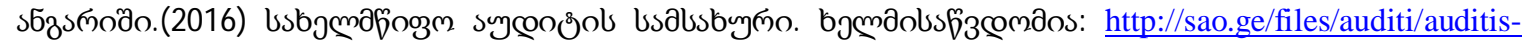
angarishebi/2016/ganaTlebis-xarisxis-uzrunvelyofa-eqe.pdf

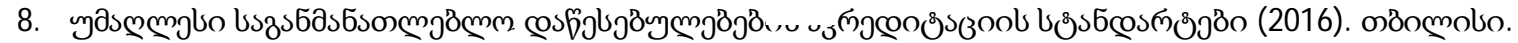

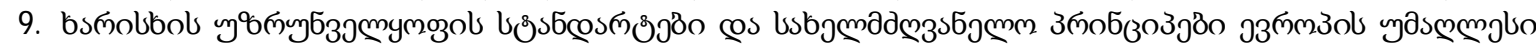

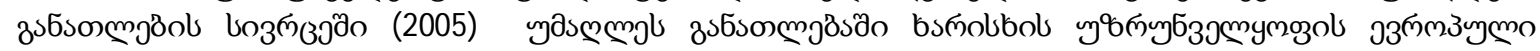
slimgoszos.

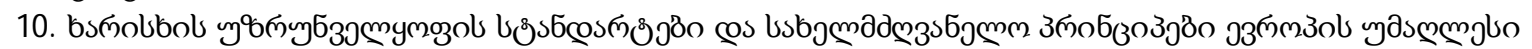

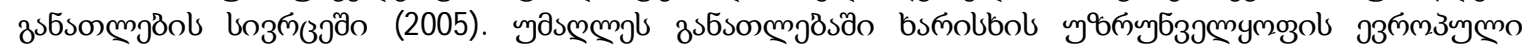
slomososos.

\title{
Legal Requirements and Procedures for the Establishment and Sustainability of the Educational Space of Georgian Universities
}

\author{
Gigilashvili Nino \\ lakob Gogebashvili Telavi State University, Telavi \\ Tatulishvili Salome \\ lakob Gogebashvili Telavi State University, Telavi
}

\begin{abstract}
Georgia's involvement in the Bologna process has completely changed and redefined the orientation of its development and, therefore, its strategic goals. Initially, the Law of Georgia on Higher Education was drafted (adopted December 21, 2004) which regulates the process of carrying out educational and scientific-research activities by higher education institutions in Georgia, and the principles and procedures of administering and financing higher education; it also defines the procedures for establishment, reorganization of the activities and for the liquidation of all the higher education institutions, as well as principles of the authorization and accreditation of higher education institutions. The given law has set minimum standards and requirements for the establishment of higher education institutions in the education system of Georgia. On the whole, the changes made and defined in the Law on Higher Education has laid the foundation for the authorization and accreditation of higher education institutions as an essential requirement for their existence and sustainability in the common European educational area.
\end{abstract}

Keywords: University, authorization, accreditation, education, law, reform 